\title{
Crystal-field interaction and oxygen stoichiometry effects in strontium-doped rare-earth cobaltates
}

\author{
A. Furrer, ${ }^{1,}{ }^{*}$ A. Podlesnyak, ${ }^{2}$ M. Frontzek, ${ }^{1}$ I. Sashin, ${ }^{3}$ J. P. Embs, ${ }^{1}$ E. Mitberg, ${ }^{4}$ and E. Pomjakushina ${ }^{5}$ \\ ${ }^{1}$ Laboratory for Neutron Scattering, Paul Scherrer Institut, CH-5232 Villigen PSI, Switzerland \\ ${ }^{2}$ Quantum Condensed Matter Division, Oak Ridge National Laboratory, Oak Ridge, Tennessee 37831-6473, USA \\ ${ }^{3}$ Joint Institute for Nuclear Research, 141980 Dubna, Moscow Region, Russia \\ ${ }^{4}$ Institute of Solid State Chemistry RAS, 620219 Ekaterinburg GSP-145, Russia \\ ${ }^{5}$ Laboratory for Developments and Methods, Paul Scherrer Institut, CH-5232 Villigen PSI, Switzerland
}

(Received 30 May 2014; revised manuscript received 13 August 2014; published 26 August 2014)

\begin{abstract}
Inelastic neutron scattering was employed to study the crystal-field interaction in the strontium-doped rare-earth compounds $R_{x} \mathrm{Sr}_{1-x} \mathrm{CoO}_{3-z}(R=\mathrm{Pr}$, Nd, Ho, and Er). Particular emphasis is laid on the effect of oxygen deficiencies that naturally occur in the synthesis of these compounds. The observed energy spectra are found to be the result of a superposition of crystal fields with different nearest-neighbor oxygen coordination at the $R$ sites. The experimental data are interpreted in terms of crystal-field parameters, which behave in a consistent manner through the rare-earth series, thereby allowing a reliable extrapolation for rare-earth ions not considered in the present work.
\end{abstract}

DOI: 10.1103/PhysRevB.90.064426

PACS number(s): 71.70.Ch, 75.47.Lx, 78.70.Nx

\section{INTRODUCTION}

Rare-earth cobaltates of the perovskite type $R \mathrm{CoO}_{3}(R=$ rare earth) have attracted much interest due to their rich phase diagrams involving structural, magnetic, spin-state, and metalinsulator transitions [1-5], as well as due to their technological applications as oxidation catalysts, electrode materials, and oxygen-permeable membranes [6-8]. More recent research has focused on hole-doped cobaltates $R_{x} A_{1-x} \mathrm{CoO}_{3}$, with $A=\mathrm{Sr}$ [9] and $A=\mathrm{Ca}$ [10], which, e.g., revealed the existence of spin-state polarons for $R=\mathrm{La}$ and $A=\mathrm{Sr}[11,12]$. The magnetic properties of these compounds depend on the crystalfield and exchange interactions of the $R$ and Co ions. While the interactions associated with the Co ions are well documented in the literature [13,14], not much is known about the interactions associated with the $R$ ions. Often some numerical data are extrapolated from other perovskites, such as $R \mathrm{NiO}_{3}$ [15] and $R \mathrm{GaO}_{3}[16,17]$, but this approach is highly questionable due to the variations of the charge distributions for different transition metal ions. In order to shed light on the crystal-field interaction at the $R$ sites, we performed inelastic neutron scattering (INS) experiments for the compounds $R_{x} \mathrm{Sr}_{1-x} \mathrm{CoO}_{3-z}(R=\mathrm{Pr}, \mathrm{Nd}$, Ho, and Er; $0.10 \leqslant x \leqslant 0.15 ; 0.23 \leqslant z \leqslant 0.30)$.

The compound $\mathrm{SrCoO}_{3}$ crystallizes in a simple cubic structure with space group $P m-3 m$ [18]. For $R_{x} \mathrm{Sr}_{1-x} \mathrm{CoO}_{3-z}$, the partial replacement of $\mathrm{Sr}^{2+}$ ions by $R^{3+}$ ions induces spin-state transitions for part of the Co ions from highspin $\mathrm{Co}^{4+}$ to low-spin $\mathrm{Co}^{3+}$ as well as oxygen deficiencies $z$. The latter lead to structural modifications, which have been examined in detail, e.g., for $R=$ Ho with $x=0.1$ and $0.15 \leqslant z \leqslant 0.49$ [19]. These details, however, are irrelevant for the present crystal-field study, since the local symmetry at the $R$ sites remains essentially cubic, as verified in INS experiments performed for $\operatorname{Pr}_{0.2} \mathrm{Sr}_{0.8} \mathrm{CoO}_{3-z}$ [20], $\mathrm{Ho}_{0.1} \mathrm{Sr}_{0.9} \mathrm{CoO}_{3-z}$ [20], and $\mathrm{Pr}_{0.5} \mathrm{Sr}_{0.9} \mathrm{CoO}_{3}$ [21]. The oxygen nonstoichiometry results in a superposition of crystal fields due to different oxygen coordinations at the $R^{3+}$ sites, as most

*Corresponding author: albert.furrer@psi.ch convincingly demonstrated, e.g., for the perovskite cuprates $\mathrm{ErBa}_{2} \mathrm{Cu}_{3} \mathrm{O}_{7-z}(0<z<1)$ [22]. The superposition of crystal fields produces both additional lines and line broadening in the observed energy spectra; thus, a proper identification of lines is important for a reliable data analysis. In fact, the conclusions reached in INS experiments carried out so far for some $R_{x} \mathrm{Sr}_{1-x} \mathrm{CoO}_{3-z}$ compounds [20,21] suffer from an incorrect line assignment and thereby from an incorrect parameterization of the crystal-field interaction. Here, we show that these problems can be overcome by supporting model calculations of the oxygen stoichiometry effects as well as by a systematic investigation over the whole rare-earth series.

The present work is organized as follows. The experimental procedure is described in Sec. II, followed in Sec. III by a summary of the underlying theoretical background. The experimental results and their analyses are presented in Sec. IV. Finally, some conclusions are given in Sec. V.

\section{EXPERIMENTAL}

\section{A. Sample synthesis}

The $R_{x} \mathrm{Sr}_{1-x} \mathrm{CoO}_{3-z}$ samples were prepared by a conventional solid state reaction using appropriate amounts of rare-earth oxides, $\mathrm{SrCO}_{3}$ and $\mathrm{Co}_{3} \mathrm{O}_{4}$, with minimum purity of $99.99 \%$. The oxygen deficiency $z$ was determined by thermogravimetric hydrogen reduction with a precision of $d z= \pm 0.01$ [23]. The single-phase character of the samples was confirmed by $\mathrm{x}$-ray powder diffraction.

\section{B. INS experiments}

The INS experiments were carried out with the use of three spectrometers in order to cover different ranges of energy transfers $\Delta E$. A well-suited apparatus for large energy transfers is the inverted geometry time-of-flight (TOF) spectrometer KDSOG at the pulsed reactor IBR-2 of the Joint Institute for Nuclear Research, Dubna, Russia. The energy of the scattered neutrons was $4.8 \mathrm{meV}$, as determined by $\mathrm{Zn}$ analyzers behind a Be filter cooled down to $T=80 \mathrm{~K}$, yielding an energy resolution of about $5 \%$ with respect to $\Delta E$. The 
INS spectra were measured at seven scattering angles between $30^{\circ}$ and $90^{\circ}$. The samples were placed into a closed-cycle dilution refrigerator yielding $T>10 \mathrm{~K}$. Inelastic neutron scattering experiments at smaller energy transfers were carried out at the Swiss Spallation Neutron Source (SINQ) of the Paul Scherrer Institut (PSI), Villigen, Switzerland, and the Spallation Neutron Source (SNS) of the Oak Ridge National Laboratory, Oak Ridge, Tennessee, USA, with use of the high-resolution TOF spectrometers FOCUS [24] and cold neutron chopper spectrometer (CNCS) [25], respectively, yielding energy resolutions for neutron energy-loss processes of typically $1-2 \%$ with respect to $\Delta E$. The samples were enclosed in Al cylinders (with a $12 \mathrm{~mm}$ diameter and $45 \mathrm{~mm}$ height) and mounted in He cryostats to achieve temperatures $T>1.5 \mathrm{~K}$. Additional experiments were performed for the empty container as well as for vanadium to allow the correction of the raw data with respect to background, detector efficiency, absorption, and detailed balance according to standard procedures.

\section{THEORETICAL BACKGROUND}

For the compounds $R_{x} \mathrm{Sr}_{1-x} \mathrm{CoO}_{3-z}$, the dominant contribution to the crystal-field interaction at the $R^{3+}$ site $(0,0,0)$ results from the nearest-neighboring oxygen ions located at positions $( \pm a / 2, \pm a / 2,0),( \pm a / 2,0, \pm a / 2)$, and $(0, \pm a / 2, \pm a / 2)$, where $a$ is the lattice parameter. For $z=$ 0 , all 12 positions are occupied by oxygen ions, but for $z>0$, some of these positions are vacant. There is a clear preference for the location of the oxygen vacancies as concluded from the observation of superstructures in the neutron diffraction patterns of $\mathrm{Ho}_{x} \mathrm{Sr}_{1-x} \mathrm{CoO}_{3-z}$ [19]. Oxygen is mainly lost from the basal planes of the octahedra around the cobalt ions, whereas the apical oxygen positions remain unaffected. Figure 1 shows the fraction of different oxygen coordinations around the $R^{3+}$ ions as a function of the oxygen deficiency $z$. The results were obtained from a Monte Carlo study in a three-dimensional lattice of $N \times N \times N$ unit cells with $N=15$. The Monte Carlo simulations were performed for $5 \leqslant N \leqslant 20$, and convergence occurred for $N \geqslant 10$. Since all compounds investigated in the present work have oxygen deficiencies $0.2<z \leqslant 0.3$, the deviations from the full 12-fold oxygen coordination are substantial, so that the observed energy spectra have to be analyzed as a superposition of crystal fields with different oxygen coordination around the $R^{3+}$ sites.

For full 12-fold oxygen coordination, the crystal-field Hamiltonian has cubic point symmetry and is given by

$$
H=B_{4}^{0} O_{4}^{0}+B_{4}^{4} O_{4}^{4}+B_{6}^{0} O_{6}^{0}+B_{6}^{4} O_{6}^{4},
$$

where the 4-fold symmetry axis is taken as polar axis. $B_{n}^{m}$ denotes the crystal-field parameters, and $O_{n}^{m}$ represents the Stevens operator equivalents [26]. The parameters $B_{n}^{m}$ can be described by the product of a charge term $A_{n}$ and a geometrical coordination factor $f_{n}^{m}$ defined by Hutchings [27]:

$$
B_{n}^{m}=A_{n} f_{n}^{m} .
$$

For cubic symmetry, we have the relations $B_{4}^{4}=5 B_{4}^{0}$ and $B_{6}^{4}=-21 B_{6}^{0}$; thus we are left with only two independent fourth- and sixth-order crystal-field parameters in Eq. (1). Consequently, the observation of two crystal-field transitions

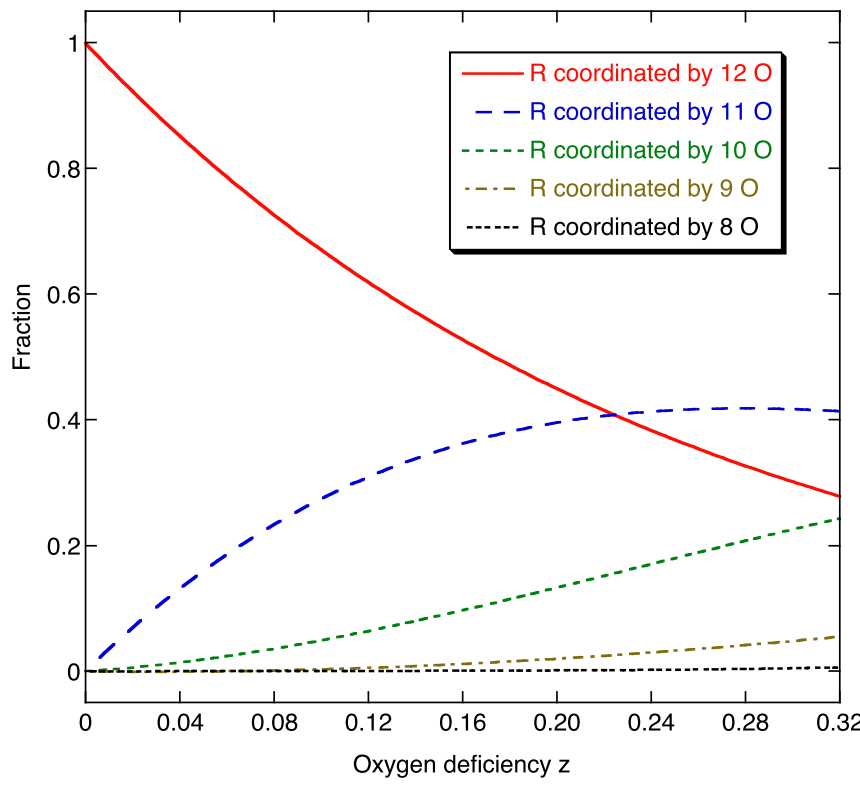

FIG. 1. (Color online) Fraction of different oxygen coordinations around the $R^{3+}$ ions in $R_{x} \mathrm{Sr}_{1-x} \mathrm{CoO}_{3-z}$ as a function of the oxygen deficiency $z$. The results were obtained from a Monte Carlo simulation in an $N \times N \times N$ lattice of unit cells with $N=15$.

(i.e., their energies and intensities) is sufficient to unambiguously determine the crystal-field parameters, which can also be estimated on the basis of a simple point-charge model involving the nearest-neighboring oxygen coordination shell:

$$
A_{n}=q|e| \chi_{n}\left\langle r^{n}\right\rangle \text {. }
$$

Here, $q$ is the charge of the oxygen ions, $e$ is the charge of the electron, $\chi_{n}$ is a reduced matrix element [27], and $\left\langle r^{n}\right\rangle$ is the $n$th moment of the radial distribution of the $4 f$ electrons [28]. For (pseudo-)cubic perovskites, the parameters calculated from Eq. (3) usually predict the correct crystal-field ground state as well as the correct sequence of crystal-field states, apart from a scaling factor for the overall crystal-field splitting.

For $R^{3+}$ ions coordinated by less than 12 oxygen ions, the point symmetry is lowered, and additional terms enter Eq. (1):

$$
H=\sum_{m=0}^{2} B_{2}^{m} O_{2}^{m}+\sum_{m=0}^{4} B_{4}^{m} O_{4}^{m}+\sum_{m=0}^{6} B_{6}^{m} O_{6}^{m} .
$$

The large number of independent crystal-field parameters makes the parameterization of crystal-field spectra somewhat arbitrary. However, as repeatedly shown in the past for several perovskite compounds [15-17,29,30], the crystal-field parameters $B_{n}^{m}$ for a particular degree $n$ can reasonably be determined by taking account of the nearest-neighboring coordination polyhedron:

$$
B_{n}^{m}=B_{n}^{0} \frac{f_{n}^{m}}{f_{n}^{0}},
$$

so that the number of independent crystal-field parameters in Eq. (4) is reduced to three. The geometrical coordination factors $f_{n}^{m}$ used in the present work are listed in Table I. 
TABLE I. Geometrical coordination factors $f_{n}^{m}$ for 12,11 , and 10 nearest-neighbor oxygen ions around the $R^{3+}$ site in the $R_{x} \mathrm{Sr}_{1-x} \mathrm{CoO}_{3-z}$ compounds. The lattice parameter was set at $a=$ $3.84 \AA$ A. For the $f_{n}^{m}(11)$ values, the particular choice of the vacant oxygen position is irrelevant. The $f_{n}^{m}(10)$ values were obtained from two vacant oxygen positions $( \pm a / 2,0, a / 2)$. Other choices of vacant oxygen positions result in slight energy shifts of the order of $10 \%$.

\begin{tabular}{ccccc}
\hline \hline$n$ & $m$ & $f_{n}^{m}(12)$ & $f_{n}^{m}(11)$ & $f_{n}^{m}(10)$ \\
\hline 2 & 0 & 0 & $-0.62440 \times 10^{-2}$ & $-0.12488 \times 10^{-1}$ \\
2 & 1 & 0 & $0.74928 \times 10^{-1}$ & 0 \\
2 & 2 & 0 & $-0.18732 \times 10^{-1}$ & $-0.37464 \times 10^{-1}$ \\
4 & 0 & $-0.14821 \times 10^{-2}$ & $-0.11380 \times 10^{-2}$ & $-0.79396 \times 10^{-3}$ \\
4 & 1 & 0 & $0.10586 \times 10^{-2}$ & 0 \\
4 & 2 & 0 & $-0.26465 \times 10^{-2}$ & $-0.52931 \times 10^{-2}$ \\
4 & 3 & 0 & $0.74103 \times 10^{-2}$ & 0 \\
4 & 4 & $-0.74103 \times 10^{-2}$ & $-0.83366 \times 10^{-2}$ & $-0.92629 \times 10^{-2}$ \\
6 & 0 & $-0.13999 \times 10^{-3}$ & $-0.13147 \times 10^{-3}$ & $-0.12294 \times 10^{-3}$ \\
6 & 1 & 0 & $-0.26384 \times 10^{-3}$ & 0 \\
6 & 2 & 0 & $-0.23557 \times 10^{-4}$ & $-0.47114 \times 10^{-4}$ \\
6 & 3 & 0 & $0.47114 \times 10^{-3}$ & 0 \\
6 & 4 & $0.29399 \times 10^{-2}$ & $0.26855 \times 10^{-2}$ & $0.24311 \times 10^{-2}$ \\
6 & 5 & 0 & $0.62190 \times 10^{-3}$ & 0 \\
6 & 6 & 0 & $-0.51825 \times 10^{-4}$ & $-0.10365 \times 10^{-3}$ \\
\hline \hline
\end{tabular}

Equations (3) and (5) are well suited to extrapolate the crystal-field parameters from a particular rare-earth compound with $R_{1}$ ions to another isostructural compound with $R_{2}$ ions through the relation [29]

$$
A_{n}\left(R_{2}\right)=A_{n}\left(R_{1}\right) \frac{\chi_{n}\left(R_{2}\right) \cdot\left\langle r^{n}\right\rangle\left(R_{2}\right)}{\chi_{n}\left(R_{1}\right) \cdot\left\langle r^{n}\right\rangle\left(R_{1}\right)} .
$$

As proven for the rare-earth series of copper-oxide perovskites [30], Eq. (6) predicts the fourth- and sixth-order crystal-field parameters with a precision of typically $10 \%$.

Figure 2 exemplifies the application of Eqs. (1)-(5) for $\mathrm{Er}_{x} \mathrm{Sr}_{1-x} \mathrm{CoO}_{3-z}$ by comparing the crystal field at the $\mathrm{Er}^{3+}$ site for 12,11 , and 10 coordinating oxygen ions. The major effect of the latter two cases is the splitting of all the quartets $\Gamma_{8}^{(n)}$ into two doublets. Identical charge parameters $A_{4}$ and $A_{6}$ are used for all cases. The second-order charge parameter $A_{2}$, which vanishes for the case of full 12-fold oxygen coordination, is seen to have little effect on the overall energy level sequence, except for the splitting of the two lowest crystal-field states. In fact, this splitting turns out to be important for an unambiguous determination of $A_{2}$ from the experimental data, since Eq. (3) cannot be applied to second-order crystal-field parameters [29].

\section{RESULTS AND DATA ANALYSIS}

$$
\text { A. } \mathrm{Er}_{x} \mathrm{Sr}_{1-x} \mathrm{CoO}_{3-z}
$$

The results of INS experiments performed for $\mathrm{Er}_{0.15} \mathrm{Sr}_{0.85} \mathrm{CoO}_{2.71}$ with use of the TOF spectrometer KDSOG are shown in Fig. 3, together with data taken for the isostructural, nonmagnetic compound $\mathrm{La}_{0.15} \mathrm{Sr}_{0.85} \mathrm{CoO}_{2.71}$, which demonstrates the absence of phonon scattering in the considered range of energy transfers. The $\mathrm{Er}_{0.15} \mathrm{Sr}_{0.85} \mathrm{CoO}_{2.71}$ data are characterized by a strong asymmetric peak at

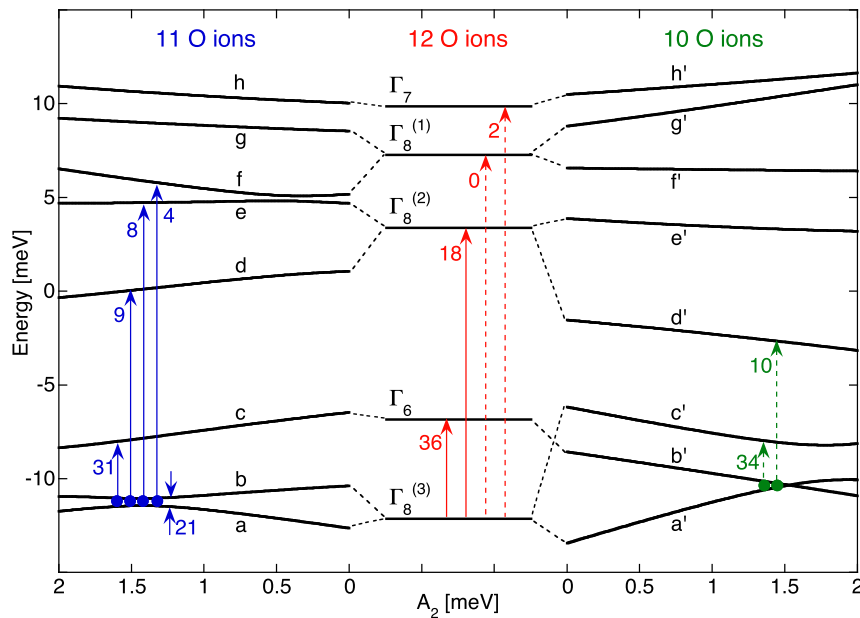

FIG. 2. (Color online) Crystal-field level sequence at the $\mathrm{Er}^{3+}$ sites in $\mathrm{Er}_{x} \mathrm{Sr}_{1-x} \mathrm{CoO}_{3-z}$ for 12, 11, and 10 coordinating $\mathrm{O}^{2-}$ ions, calculated from Eqs. (1)-(5) with $A_{4}=0.306 \mathrm{meV}$ and $A_{6}=$ $0.0151 \mathrm{meV}$. The arrows mark selected ground-state transitions, and the corresponding numbers denote the transition probability $\left|\left\langle\Gamma_{n}\left|J_{\perp}\right| \Gamma_{m}\right\rangle\right|^{2}$.

$2<\Delta E<7 \mathrm{meV}$ and a double-peak structure at $10<$ $\Delta E<20 \mathrm{meV}$. Additional experiments were carried out for $\mathrm{Er}_{0.10} \mathrm{Sr}_{0.90} \mathrm{CoO}_{2.70}$ at low energy transfers with use of the TOF spectrometer FOCUS as displayed in Fig. 4, which shows the existence of a partly resolved line peaking at $\Delta E \approx 0.3 \mathrm{meV}$. All these features cannot be explained on the basis of the "ideal" crystal-field Hamiltonian Eq. (1), but the analysis of the observed energy spectra has to be based on a superposition of crystal fields with different oxygen coordination around the $\mathrm{Er}^{3+}$ ions as outlined in Sec. III.

For an oxygen deficiency $z=0.29$ and a statistical distribution of the oxygen vacancies, we expect the observed energy spectra to be a superposition of crystal fields resulting from 12-fold, 11-fold, and 10-fold oxygen coordination with

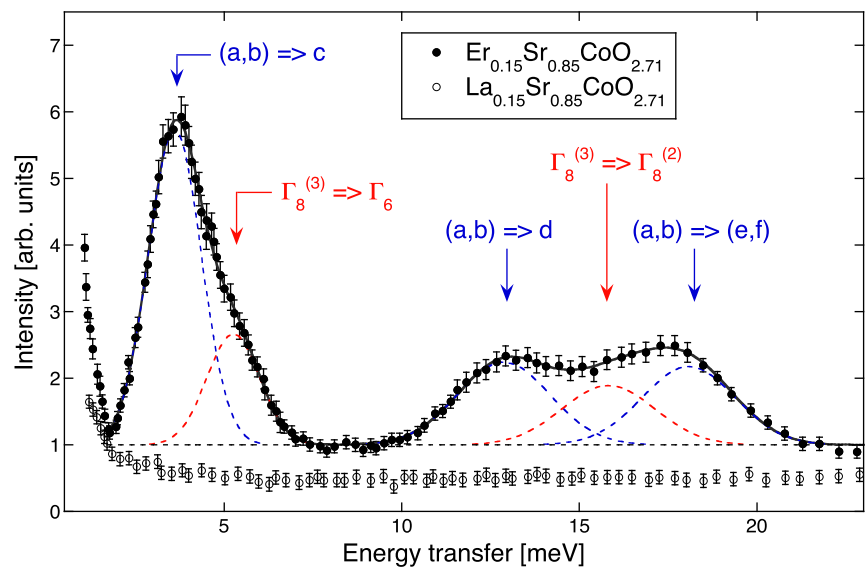

FIG. 3. (Color online) Energy spectra of neutrons scattered from $\mathrm{Er}_{0.15} \mathrm{Sr}_{0.85} \mathrm{CoO}_{2.71}$ and $\mathrm{La}_{0.15} \mathrm{Sr}_{0.85} \mathrm{CoO}_{2.71}$ at $T=10 \mathrm{~K}$. The data were taken with the use of the TOF spectrometer KDSOG for moduli of the scattering vector $1.6<Q<3.3 \AA^{-1}$. The lines denote Gaussian fits as explained in the text. The arrows mark the crystal-field transitions according to Fig. 2. 


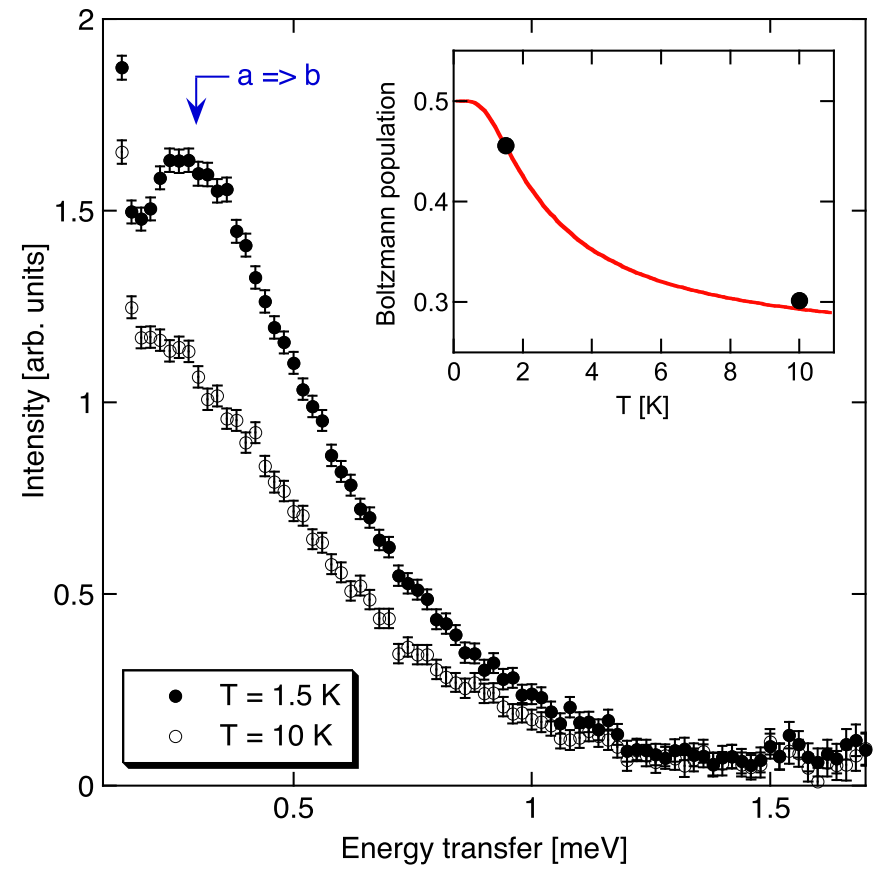

FIG. 4. (Color online) Energy spectra of neutrons scattered from $\mathrm{Er}_{0.10} \mathrm{Sr}_{0.90} \mathrm{CoO}_{2.70}$. The data were taken with the use of the TOF spectrometer FOCUS for moduli of the scattering vector $0.4<$ $Q<2.2 \AA^{-1}$. The incoming neutron energy was $3.3 \mathrm{meV}$. The arrow marks the transition according to Fig. 2. The inset shows the temperature dependence of the intensities integrated in the range $0.2 \leqslant \Delta E \leqslant 0.8 \mathrm{meV}$ (spheres) and scaled to the calculated Boltzmann population factor for the ground-state doublet (line).

approximate weights of $30 \%, 40 \%$, and $20 \%$, respectively (neglecting the small fraction of $\mathrm{Er}^{3+}$ ions with 9-fold and 8 -fold oxygen coordination; see Fig. 1). For 12-fold oxygen coordination, we derive from Eqs. (1) and (3) the quartet $\Gamma_{8}^{(3)}$ as the ground state, and the excited crystal-field states are-with increasing energy separation - the doublet $\Gamma_{6}$, the quartets $\Gamma_{8}^{(2)}$ and $\Gamma_{8}^{(1)}$, and the doublet $\Gamma_{7}$. The corresponding crystal-field level sequence, properly scaled to the observed energy spectra, is displayed in the middle of Fig. 2, which predicts the presence of two strong ground-state transitions $\Gamma_{8}^{(3)} \rightarrow \Gamma_{6}$ and $\Gamma_{8}^{(3)} \rightarrow \Gamma_{8}^{(2)}$, whereas the transitions to the two highest states, $\Gamma_{8}^{(1)}$ and $\Gamma_{7}$, could not be resolved in the experiments because of their small transition probabilities. Statistically, of equal importance are the crystal-field contributions for 11 -fold and 10-fold oxygen coordination, as displayed in
Fig. 2. Obviously, the observed low-energy line peaking at $\Delta E \approx 0.3 \mathrm{meV}$ (see Fig. 4) has to be associated with the ground-state doublet-doublet splittings $a \rightarrow b$ and $a^{\prime} \rightarrow b^{\prime}$, which fix the second-order crystal-field parameter at $A_{2}=$ 1.4(2) $\mathrm{meV}$. This interpretation is supported by the temperature dependence of the intensities, as shown in the inset of Fig. 4. The strong asymmetric peak at $2<\Delta E<7 \mathrm{meV}$ results then from the ground-state transitions $\Gamma_{8}^{(3)} \rightarrow \Gamma_{6},(a, b) \rightarrow c$, and $\left(a^{\prime}, b^{\prime}\right) \rightarrow c^{\prime}$ with continuously decreasing energies, but a least-squares Gaussian fit allows a decomposition into only two lines (see Fig. 3). Moreover, for 10-fold coordination, the model predicts a strong ground-state transition $\left(a^{\prime}, b^{\prime}\right) \rightarrow d^{\prime}$ at $\Delta E \approx 7.5 \mathrm{meV}$, which, however, was not observed in the experiments (see Fig. 3). Obviously, the oxygen vacancies are not statistically distributed, but there is a strong preference for 11 -fold coordination. We therefore interpret the energy spectra as a superposition of crystal fields with 12-fold and 11-fold oxygen coordination. The double-peak structure at $10<\Delta E$ $<20 \mathrm{meV}$ is then composed of three transitions, which were least-squares fitted by Gaussian lines. The assignment of the transitions is indicated by arrows in Fig. 3. From the observed intensities and considering the transition probabilities shown in Fig. 2, we conclude that 25(5)\% of the $\mathrm{Er}^{3+}$ ions have an ideal 12 -fold oxygen coordination, whereas $75(5) \%$ of the $\mathrm{Er}^{3+}$ ions are coordinated by 11 (and fewer) oxygen ions. The resulting crystal-field parameters are listed in Table II.

\section{B. $\mathrm{Ho}_{0.10} \mathrm{Sr}_{0.90} \mathrm{CoO}_{2.73}$}

Energy spectra observed for $\mathrm{Ho}_{0.10} \mathrm{Sr}_{0.90} \mathrm{CoO}_{2.73}$ with use of the TOF spectrometers FOCUS and KDSOG are shown in Figs. 5(a) and 5(b), respectively, which are complemented by high-resolution experiments performed on the TOF spectrometer CNCS displayed in Fig. 6. All these data are characterized by strong ground-state transitions centered at 1,3 , and $6 \mathrm{meV}$, as well as by weak transitions around 15 and $20 \mathrm{meV}$, which were least-squares fitted by Gaussian lines as shown in Fig. 5.

For an oxygen deficiency $z=0.27$, the data analysis has to be based on a superposition of crystal fields with different oxygen coordination, similar to the case of $\mathrm{Er}_{x} \mathrm{Sr}_{1-x} \mathrm{CoO}_{2.71}$ discussed in Sec. IV A. For 12-fold oxygen coordination, the crystal-field states at the $\mathrm{Ho}^{3+}$ site comprise four triplets $\Gamma_{4}^{(1)}, \Gamma_{4}^{(2)}, \Gamma_{5}^{(1)}$, and $\Gamma_{5}^{(2)}$, two doublets $\Gamma_{3}^{(1)}$ and $\Gamma_{3}^{(2)}$, and a singlet $\Gamma_{1}$, which are completely split into 17 singlet states for 11-fold oxygen coordination (and similarly for 10-fold oxygen coordination). However, these splittings could not by resolved in the experiments, but they give rise to a substantial broadening of the observed transitions as visualized in Figs. 5

TABLE II. Crystal-field parameters $A_{n}$ of the $R_{x} \mathrm{Sr}_{1-x} \mathrm{CoO}_{3-z}$ compounds. The parameters $B_{n}^{m}=A_{n} f_{n}^{m}$ (Eq. (2)) can be calculated with use of the geometrical coordination factors $f_{n}^{m}$ listed in Table I.

\begin{tabular}{|c|c|c|c|c|}
\hline Compound & $A_{2}[\mathrm{meV}]$ & $A_{4}[\mathrm{meV}]$ & $A_{6}[\mathrm{meV}]$ & Remarks \\
\hline $\mathrm{Er}_{0.15} \mathrm{Sr}_{0.85} \mathrm{CoO}_{2.71}$ & $1.4(2)$ & $0.306(27)$ & $1.51(14) \times 10^{-2}$ & Determined from Figs. 3 and 4 \\
\hline $\mathrm{Ho}_{0.10} \mathrm{Sr}_{0.90} \mathrm{CoO}_{2.73}$ & & $-0.233(19)$ & $-8.57(34) \times 10^{-3}$ & Determined from Figs. 5 and 6 \\
\hline $\mathrm{Pr}_{0.10} \mathrm{Sr}_{0.90} \mathrm{CoO}_{2.77}$ & & -14.2 & 1.79 & Extrapolated from $\mathrm{Er}_{0.15} \mathrm{Sr}_{0.85} \mathrm{CoO}_{2.71}$ \\
\hline $\mathrm{Pr}_{0.10} \mathrm{Sr}_{0.90} \mathrm{CoO}_{2.77}$ & $-20(4)$ & & & Determined from Fig. 7 \\
\hline $\mathrm{Nd}_{0.10} \mathrm{Sr}_{0.90} \mathrm{CoO}_{2.74}$ & & -4.7 & -0.89 & Extrapolated from $\mathrm{Er}_{0.15} \mathrm{Sr}_{0.85} \mathrm{CoO}_{2.71}$ \\
\hline $\mathrm{Nd}_{0.10} \mathrm{Sr}_{0.90} \mathrm{CoO}_{2.74}$ & $-4.5(9)$ & & & Determined from Fig. 9 \\
\hline
\end{tabular}




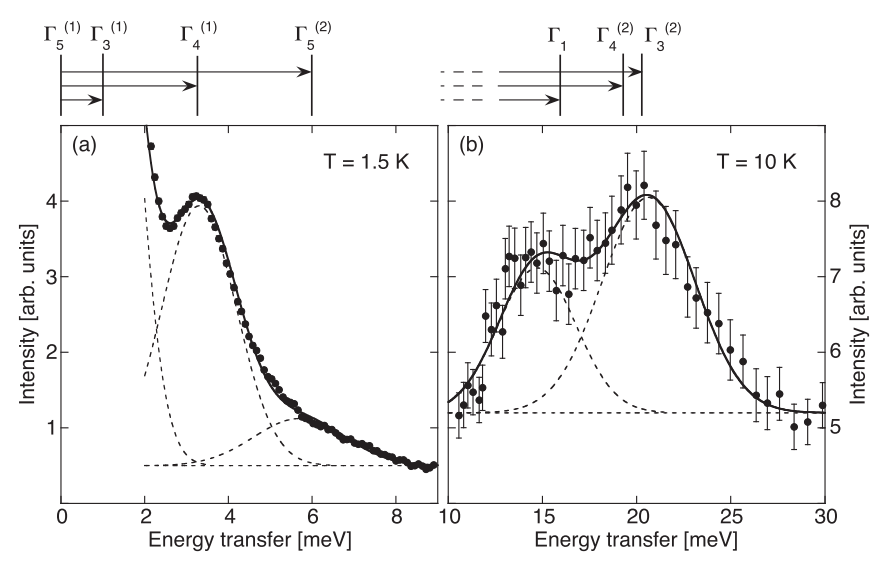

FIG. 5. Energy spectra of neutrons scattered from $\mathrm{Ho}_{0.10} \mathrm{Sr}_{0.90} \mathrm{CoO}_{2.73}$. The lines denote Gaussian fits as explained in the text. The top displays the crystal-field level sequence derived from the data, and the arrows mark the observed transitions. (a) Data taken at $T=1.5 \mathrm{~K}$ with the use of the TOF spectrometer FOCUS for moduli of the scattering vector $0.5<Q<4.9 \AA^{-1}$. The incoming neutron energy was $15.5 \mathrm{meV}$. (b) Data taken at $T=10 \mathrm{~K}$ with the use of the TOF spectrometer KDSOG for moduli of the scattering vector $2.4<Q<3.7 \AA^{-1}$.

and 6. We therefore treat the observed energy spectra on the basis of Eq. (1) by taking account of the additional splittings as line broadening effects. The best agreement between the observed and calculated energy spectra is obtained by the crystal-field level sequence displayed on top of Fig. 5, and the corresponding crystal-field parameters are listed in Table II. The temperature dependence of the intensities of the lowest transition $\Gamma_{5}^{(1)} \rightarrow \Gamma_{3}^{(1)}$ supports this interpretation, as shown by the inset in Fig. 6. It is interesting to note that the $\Gamma_{5}^{(1)} \rightarrow \Gamma_{1}$ transition, which is forbidden for cubic point symmetry, has

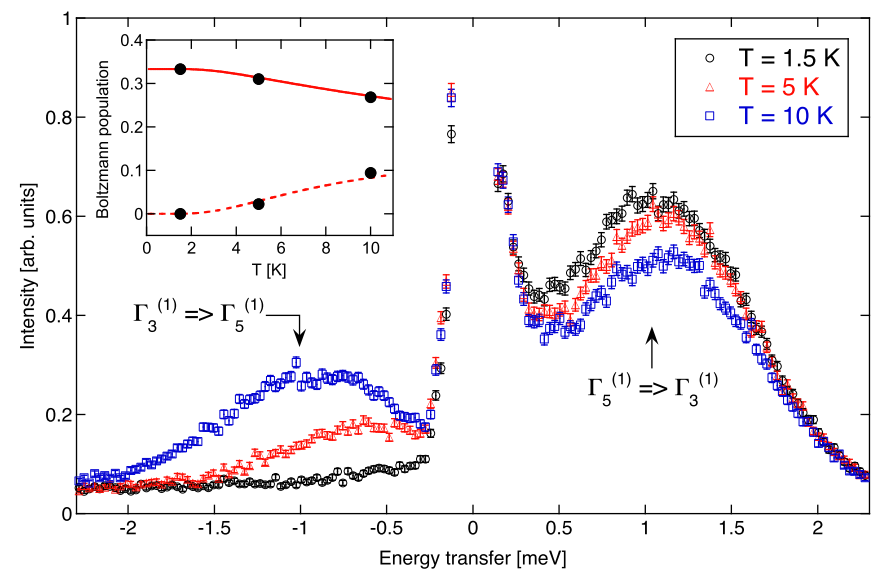

FIG. 6. (Color online) Energy spectra of neutrons scattered from $\mathrm{Ho}_{0.10} \mathrm{Sr}_{0.90} \mathrm{CoO}_{2.73}$. The data were taken with the use of the TOF spectrometer CNCS for moduli of the scattering vector $0.8<Q<1.8 \AA^{-1}$. The incoming neutron energy was $3.31 \mathrm{meV}$. The arrows mark the transitions according to Fig. 5. The inset shows the temperature dependence of the intensity integrated in the range $0.8 \leqslant|\Delta E| \leqslant 1.2$ $\mathrm{meV}$ (spheres) and scaled to the calculated Boltzmann population factor for the ground-state triplet $\Gamma_{5}{ }^{(1)}$ (full line) and the first excited doublet $\Gamma_{3}{ }^{(1)}$ (broken line).

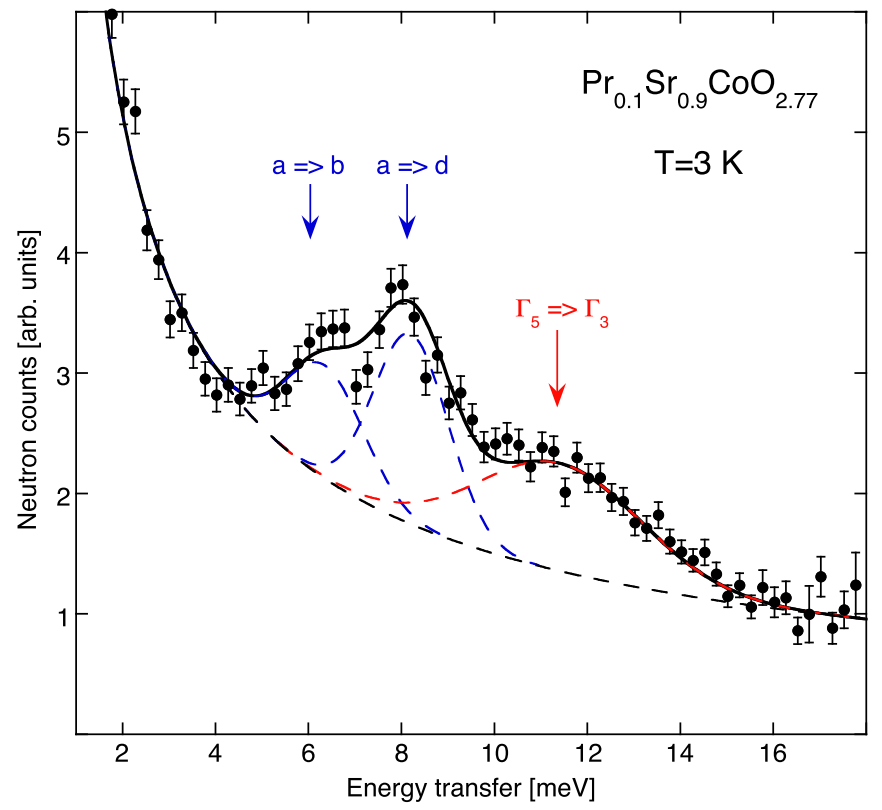

FIG. 7. (Color online) Energy spectrum of neutrons scattered from $\operatorname{Pr}_{0.10} \mathrm{Sr}_{0.90} \mathrm{CoO}_{2.77}$ at $T=3 \mathrm{~K}$. The data were taken with the use of the TOF spectrometer CNCS for moduli of the scattering vector $1.5<Q<2.5 \AA^{-1}$. The incoming neutron energy was $20 \mathrm{meV}$. The lines denote Gaussian fits on top of a power-law background as explained in the text. The arrows mark the crystal-field transitions according to Fig. 8.

gained intensity through the presence of $\mathrm{Ho}^{3+}$ ions with 11-fold (and 10-fold) oxygen coordination.

$$
\text { C. } \operatorname{Pr}_{0.10} \mathrm{Sr}_{0.90} \mathrm{CoO}_{2.77}
$$

An energy spectrum observed for $\operatorname{Pr}_{0.10} \mathrm{Sr}_{0.90} \mathrm{CoO}_{2.77}$ with use of the TOF spectrometer CNCS is shown in Fig. 7, which is characterized by partly resolved peaks at $\Delta E \approx 6$, 8 , and $12 \mathrm{meV}$. The data were least-squares fitted by three Gaussian lines on top of a sloping background described by the power-law $\alpha \cdot \exp (-\beta \cdot \Delta E)$. We extrapolated the crystal-field parameters from $\mathrm{Er}_{x} \mathrm{Sr}_{1-x} \mathrm{CoO}_{3-z}$ to $\mathrm{Pr}_{x} \mathrm{Sr}_{1-x} \mathrm{CoO}_{3-z}$ on the basis of Eq. (6) as listed in Table II. The resulting energy-level scheme is displayed in Fig. 8(a), which predicts the lowest ground-state transition $\Gamma_{5} \rightarrow \Gamma_{3}$ to be at $\Delta E=11.3 \mathrm{meV}$, i.e., exactly at the position of the highest energy peak observed in Fig. 7. The double-peak structure at $\Delta E \approx 7 \mathrm{meV}$ can then be associated with crystal-field transitions for 11-fold oxygen coordination as shown in Fig. 8, where the secondorder crystal-field parameter $A_{2}$ has to be adjusted to the experimental data. The best agreement is obtained for $A_{2}=$ -20(4) meV, as shown in Fig. 8(b). Excitations to crystalfield states above $20 \mathrm{meV}$ could not be observed, which is most likely due to substantial line broadening resulting from the oxygen nonstoichiometry as well as to strong phonon scattering contributions at higher energy transfers [21].

\section{D. $\mathrm{Nd}_{0.10} \mathrm{Sr}_{0.90} \mathrm{CoO}_{2.74}$}

The crystal-field parameters extrapolated from $\mathrm{Er}_{x} \mathrm{Sr}_{1-x} \mathrm{CoO}_{3-z}$ to $\mathrm{Nd}_{x} \mathrm{Sr}_{1-x} \mathrm{CoO}_{3-z}$ on the basis of Eq. (6) 


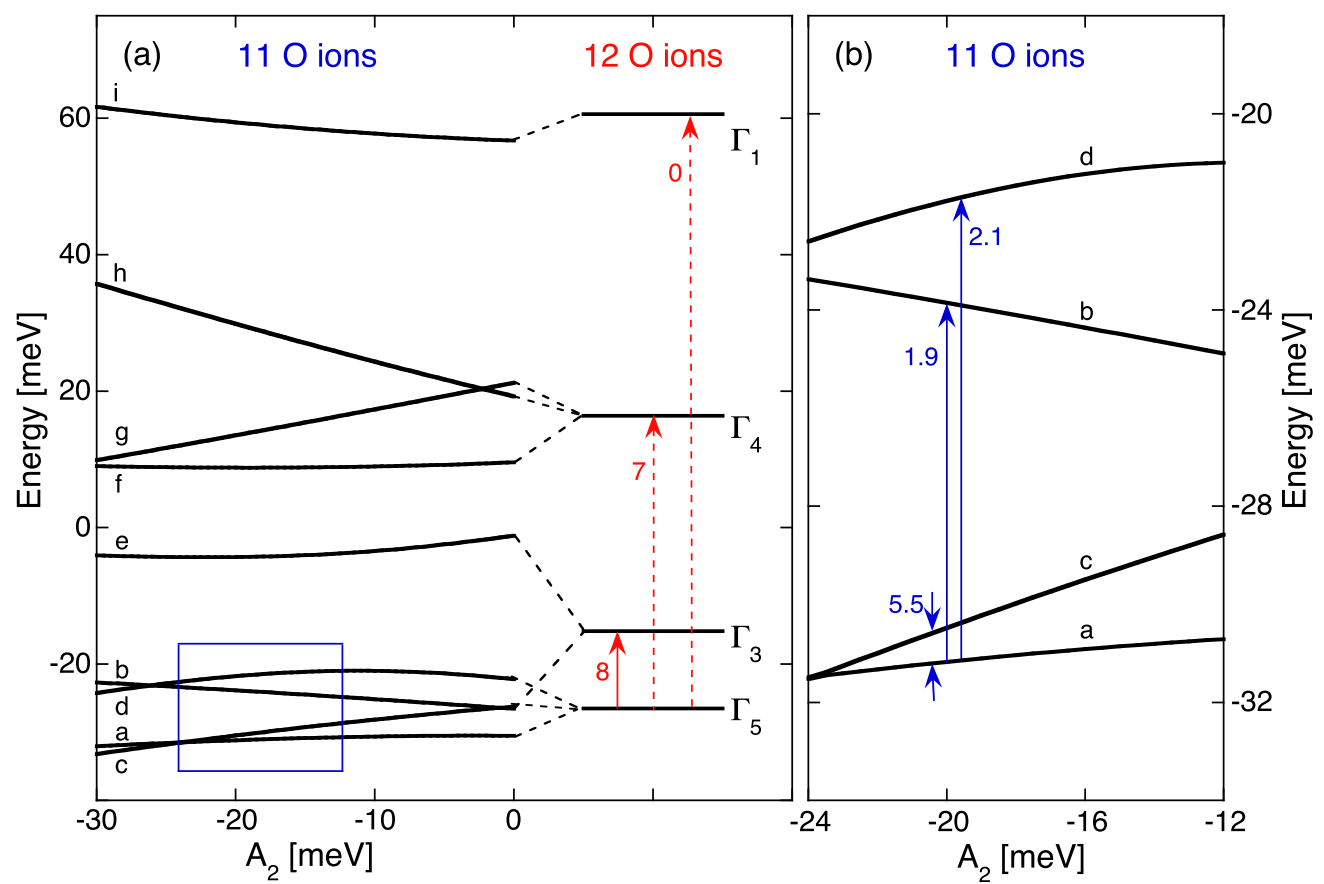

FIG. 8. (Color online) Crystal-field level sequence at the $\operatorname{Pr}^{3+}$ sites in $\operatorname{Pr}_{x} \operatorname{Sr}_{1-x} \mathrm{CoO}_{3-z}$ for 12 and 11 coordinating oxygen ions, calculated from Eqs. (1)-(5) with $A_{4}=-14.2 \mathrm{meV}$ and $A_{6}=1.79 \mathrm{meV}$. The rectangle in (a) is displayed as an enlarged figure in (b). The arrows mark selected ground-state transitions, and the corresponding numbers denote the transition probability $\left|\left\langle\Gamma_{n}\left|J_{\perp}\right| \Gamma_{m}\right\rangle\right|^{2}$.

are listed in Table II. They predict the quartet $\Gamma_{8}^{(1)}$ to be the ground state, and the excited states are the doublet $\Gamma_{6}$

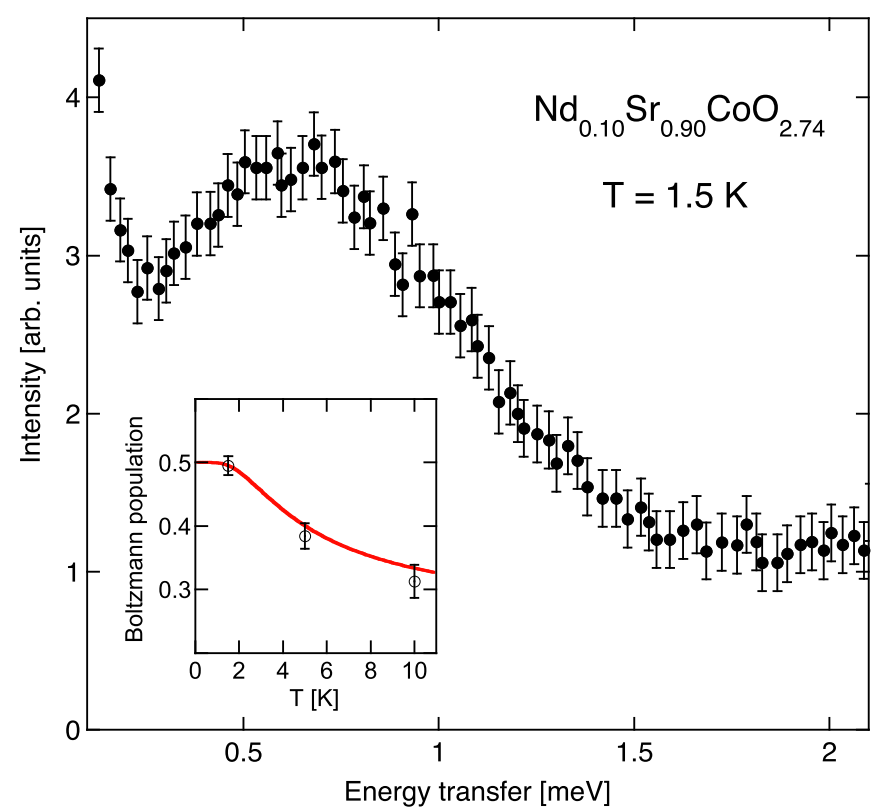

FIG. 9. (Color online) Energy spectrum of neutrons scattered from $\mathrm{Nd}_{0.10} \mathrm{Sr}_{0.90} \mathrm{CoO}_{2.74}$ at $T=1.5 \mathrm{~K}$. The data (filled circles) were taken with the use of the TOF spectrometer CNCS for moduli of the scattering vector $0.8<Q<1.8 \AA^{-1}$. The incoming neutron energy was $3 \mathrm{meV}$. The inset shows the temperature dependence of the intensity integrated in the range $0.35 \leqslant \Delta E \leqslant 1.15 \mathrm{meV}$ (open circles; data taken with the use of the TOF spectrometer FOCUS) and scaled to the calculated Boltzmann population factor for the ground-state quartet $\Gamma_{8}{ }^{(1)}$ with a doublet-doublet splitting of $0.6 \mathrm{meV}$ (line). at $17 \mathrm{meV}$ and the quartet $\Gamma_{8}^{(2)}$ at $43 \mathrm{meV}$. However, INS experiments performed for energy transfers up to $\Delta E=$ $50 \mathrm{meV}$ with the use of the TOF spectrometer CNCS failed to observe any intensity associated with crystal-field transitions for reasons given in Sec. IV C; moreover, the first excited crystal-field transition $\Gamma_{8}^{(1)} \rightarrow \Gamma_{6}$ has a rather small transition probability. Nevertheless, high-resolution INS experiments carried out with the use of the TOF spectrometers CNCS and FOCUS revealed the presence of a line at $\Delta E \approx 0.6 \mathrm{meV}$, as shown in Fig. 9. The magnetic origin of the line was confirmed by the $Q$-dependence of the intensity, which was in agreement with the square of the magnetic form factor. Its origin is most likely due to the splitting of the ground-state quartet $\Gamma_{8}^{(1)}$ into two doublets, as confirmed by the temperature dependence of the intensity shown in the inset of Fig. 9. The splitting probably results from a slight distortion of the cubic site symmetry around the $\mathrm{Nd}^{3+}$ ions and can be described by the second-order crystal-field parameter $A_{2}=-4.5(9) \mathrm{meV}$.

\section{DISCUSSION AND CONCLUSIONS}

We presented a comprehensive INS analysis of the crystalfield interactions and associated oxygen stoichiometry effects in the $R_{x} \mathrm{Sr}_{1-x} \mathrm{CoO}_{3-z}$ compounds. The systematic study including the heavy $R$ ions Er and Ho as well as the light $R$ ions $\mathrm{Pr}$ and $\mathrm{Nd}$ allowed a consistent parameterization in terms of phenomenological crystal-field parameters. The reliability of our analysis is supported by the rather good agreement of the effective charges of the nearest-neighbor coordinating oxygen ions for different $R$ ions. Based on Eq. (3), we derive from the crystal-field parameters $A_{4}$ listed in Table II consistent oxygen charges $q=-3.7(3)|e|$ and $q=-3.4(3)|e|$ for the heavy $R$ ions Er and Ho, respectively. The oxygen charges derived from 
the crystal-field parameter $A_{6}$ are equally consistent with $q=$ $-2.8(3)|e|$ and $q=-2.4(1)|e|$ for Er and Ho, respectively. Deviations from the "ideal" oxygen charge $q=-2|e|$ are expected due to the screening of the $4 f$ electrons by the outer shells, which can be taken into account by adding in Eq. (3) shielding (and antishielding) factors defined in Refs. [29,31]. For the light $R$ ions $\mathrm{Pr}$ and $\mathrm{Nd}$, we failed to detect most of the excited crystal-field transitions due to the reasons explained in Sec. IV, but the observed low-energy features were found to be in good agreement with the crystal-field parameters extrapolated from $R=$ Er.

The calculations describing the oxygen stoichiometry effects were based on Eqs. (3) and (5), which correspond to a point-charge model involving only the nearest-neighbor oxygen coordination shell. This simple model turned out to work surprisingly well, as it was restricted to the short-range fourth- and sixth-order crystal-field parameters $A_{4}$ and $A_{6}$. On the other hand, the parameterization of the long-range secondorder crystal-field term $A_{2}$ relied exclusively on particular features of the observed energy spectra.

Clementyev et al. [21] reported a significant change of the crystal-field potential upon variation of the Pr content $x$ in the $\mathrm{Pr}_{x} \mathrm{Sr}_{1-x} \mathrm{CoO}_{3-z}$ compounds. The corresponding transitions observed in INS experiments are listed in Table III. By going from $x=0.50$ to $x=0.10$, the energy of the $\Gamma_{5} \rightarrow \Gamma_{3}$ transition is drastically reduced from $15 \mathrm{meV}$ to $11.3 \mathrm{meV}$, which cannot be understood by the small lattice expansion from $a=3.83 \AA$ to $a=3.84 \AA$. However, as mentioned in Sec. I, the mutual replacement of $\mathrm{Pr}^{3+}$ and $\mathrm{Sr}^{2+}$ ions results in a change of the $\mathrm{Co}^{4+} / \mathrm{Co}^{3+}$ ratio, which obviously has substantial effects on the overall charge distribution and modifies the crystal-field potential accordingly.

Table III also supports our analysis of the observed energy spectra in terms of a superposition of crystal fields with different oxygen coordination at the $R^{3+}$ sites. Practically all the $\operatorname{Pr}^{3+}$ sites in $\operatorname{Pr}_{0.50} \mathrm{Sr}_{0.50} \mathrm{CoO}_{2.98}$ are ideally coordinated by 12 oxygen ions; thus, no lines associated with 11 -fold (and 10-fold) oxygen coordination were observed below the
TABLE III. Transitions observed in INS experiments performed for $\operatorname{Pr}_{x} \mathrm{Sr}_{1-x} \mathrm{CoO}_{3-z}$ as a function of the Pr content $x$. For the assignment of the transitions, we refer to Fig. 8.

\begin{tabular}{llcc}
\hline \hline Compound & $\Gamma_{5} \rightarrow \Gamma_{3}$ & $a \rightarrow(b, d)$ & Reference \\
\hline $\operatorname{Pr}_{0.50} \mathrm{Sr}_{0.50} \mathrm{CoO}_{2.98}$ & $15 \mathrm{meV}$ & - & 21 \\
$\mathrm{Pr}_{0.20} \mathrm{Sr}_{0.80} \mathrm{CoO}_{2.70}$ & $13 \mathrm{meV}$ & $8 \mathrm{meV}$ & 20 \\
$\mathrm{Pr}_{0.10} \mathrm{Sr}_{0.90} \mathrm{CoO}_{2.77}$ & $11.3 \mathrm{meV}$ & $7 \mathrm{meV}^{\mathrm{a}}$ & Present work \\
\hline \hline
\end{tabular}

${ }^{\text {a Corresponds to the average of the transitions } a \rightarrow b \text { and } a \rightarrow d \text { in }}$ Fig. 7.

$\Gamma_{5} \rightarrow \Gamma_{3}$ transition [21]. On the other hand, oxygen nonstoichiometry effects dominate the crystal-field interactions in $\operatorname{Pr}_{0.20} \mathrm{Sr}_{0.80} \mathrm{CoO}_{2.70}$. In fact, the line associated with the $a \rightarrow(b, d)$ transition turned out to be twice as strong as the $\Gamma_{5} \rightarrow \Gamma_{3}$ transition [20], whereas for $\operatorname{Pr}_{0.10} \mathrm{Sr}_{0.90} \mathrm{CoO}_{2.77}$ with an intermediate oxygen deficiency, the intensities of the two transitions are roughly equal.

In conclusion, our work provides a consistent basis for the parameterization of the crystal-field potential in the $\mathrm{R}_{x} \mathrm{Sr}_{1-x} \mathrm{CoO}_{3-z}$ compounds, which was experimentally verified for $R=$ Er and $R=$ Ho, but it can be reliably extrapolated for the whole rare-earth series as proven for $R=\operatorname{Pr}$ and $R=\mathrm{Nd}$. Moreover, particular emphasis was laid on oxygen nonstoichiometry effects, which influence the crystal-field potential in a decisive manner, but they can conveniently be handled by the model calculations outlined in the present work.

\section{ACKNOWLEDGMENT}

Part of this work was performed at the Swiss Spallation Neutron Source, Paul Scherrer Institut, Villigen, Switzerland. Research at the Oak Ridge National Laboratory Spallation Neutron Source Oak Ridge, Tennessee, USA, was supported by the Scientific User Facilities Division, Office of Basic Energy Sciences, U.S. Department of Energy.
[1] G. Zhang, E. Gorelov, E. Koch, and E. Pavarini, Phys. Rev. B 86, 184413 (2012).

[2] Z. Jirák, J. Hejtmánek, K. Knížek, P. Novák, E. Šantavá, and H. Fujishiro, J. Appl. Phys. 115, 17E118 (2014).

[3] B. Scherrer, A. S. Harvey, S. Tanasescu, F. Teodorescu, A. Botea, K. Conder, A. N. Grundy, J. Martynczuk, and L. J. Gauckler, Phys. Rev. B 84, 085113 (2011).

[4] J. Yu, D. Phelan, and D. Louca, Phys. Rev. B 84, 132410 (2011).

[5] J. A. Alonso, M. J. Martínez-Lope, C. de la Callea, and V. Pomjakushin, J. Mater. Chem. 16, 1555 (2006).

[6] R. H. E. van Doorn and A. J. Burggraf, Solid State Ionics 128, 65 (2000).

[7] L. Malavasi, C. Tealdi, G. Flor, G. Chiodelli, V. Cervetto, A. Montenero, and M. Borell, Sens. Actuators B 105, 407 (2005).
[8] F. Capon, A. Boileau, C. Carteret, N. Martin, P. Boulet, and J. F. Pierson, J. Appl. Phys. 114, 113510 (2013).

[9] M. James, A. Tedesco, D. Cassidy, M. Colella, and P. J. Smythe, J. Alloys Compd. 419, 201 (2006).

[10] G. J. Thorogood, P.-Y. Orain, M. Ouvry, B. Piriou, T. Tedesco, K. S. Wallwork, J. Herrmann, and M. James, Solid State Sci. 13, 2113 (2011).

[11] A. Podlesnyak, M. Russina, A. Furrer, A. Alfonsov, E. Vavilova, V. Kataev, B. Büchner, Th. Strässle, E. Pomjakushina, K. Conder, and D. I. Khomskii, Phys. Rev. Lett. 101, 247603 (2008).

[12] A. Podlesnyak, G. Ehlers, M. Frontzek, A. Furrer, Th. Strässle, E. Pomjakushina, K. Conder, F. Demmel, and D. I. Khomskii, Phys. Rev. B 83, 134430 (2011).

[13] D. Phelan, D. Louca, S. N. Ancona, S. Rosenkranz, H. Zheng, and J. F. Mitchell, Phys. Rev. B 79, 094420 (2009). 
[14] H. M. Aarbogh, J. Wu, L. Wang, H. Zheng, J. F. Mitchell, and C. Leighton, Phys. Rev. B 74, 134408 (2006).

[15] S. Rosenkranz, M. Medarde, F. Fauth, J. Mesot, M. Zolliker, A. Furrer, U. Staub, P. Lacorre, R. Osborn, R. S. Eccleston, and V. Trounov, Phys. Rev. B 60, 14857 (1999).

[16] A. Podlesnyak, S. Rosenkranz, F. Fauth, W. Marti, A. Furrer, A. Mirmelstein, and H. J. Scheel, J. Phys.: Condens. Matter 5, 8973 (1993).

[17] A. Podlesnyak, S. Rosenkranz, F. Fauth, W. Marti, H. J. Scheel, and A. Furrer, J. Phys.: Condens. Matter 6, 4099 (1994).

[18] H. L. Yakel, Jr., Acta Cryst. 8, 394 (1955).

[19] S. Streule, M. Medarde, A. Podlesnyak, E. Pomjakushina, K. Conder, S. Kazakov, J. Karpinski, and J. Mesot, Phys, Rev. B 73, 024423 (2006).

[20] A. Podlesnyak, A. Mirmelstein, N. Golosova, E. Mitberg, I. Leonidov, V. Kozhevnikov, I. Sashin, F. Altorfer, and A. Furrer, Appl. Phys. A 74, S1746 (2002). The oxygen contents of the samples were indicated to be $3 \pm 0.005$, but later re-examined to be 2.70(1) for Pr and 2.73(1) for Ho (private communication by A.P.).

[21] E. S. Clementyev, P. Alekseev, V. V. Efimov, I. O. Troyanchuk, A. S. Ivanov, V. N. Lazukov, and V. V. Sikolenko, J. Surf. Inv.: X-ray, Synchr. Neutr. Tech. 6, 553 (2012). The exact oxygen content of the sample was 2.98 (private communication by E.S.C.).

[22] J. Mesot, P. Allenspach, U. Staub, A. Furrer, and H. Mutka, Phys. Rev. Lett. 70, 865 (1993).

[23] K. Conder, E. Pomjakushina, A. Soldatov, and E. Mitberg, Mater. Res. Bull. 40, 257 (2005).

[24] S. Janssen, J. Mesot, L. Holitzner, A. Furrer, and R. Hempelmann, Physica B 234-236, 1174 (1997).

[25] G. Ehlers, A. A. Podlesnyak, J. L. Niedziela, E. B. Iverson, and P. E. Sokol, Rev. Sci. Instrum. 82, 085108 (2011).

[26] K. W. H. Stevens, Proc. Phys. Soc. A 65, 209 (1952).

[27] M. T. Hutchings, in Solid State Physics, edited by F. Seitz and D. Turnbull (Academic, New York, 1964), Vol. 16, p. 227.

[28] W. B. Lewis, in Magnetic Resonance and Related Phenomena, edited by I. Ursu (Publishing House of the Academy of Romania, Bucharest, 1971), p. 717.

[29] A. Furrer and A. Podlesnyak, in Handbook of Applied Solid State Spectroscopy, edited by D. R. Vij (Springer, New York, 2006), p. 257.

[30] J. Mesot and A. Furrer, in Neutron Scattering in Layered CopperOxide Superconductors, edited by A. Furrer (Kluwer, Dordrecht, 1998), p. 335.

[31] R. M. Sternheimer, Phys. Rev. 146, 140 (1966). 